\title{
Curvas de Crescimento na Produção Animal
}

\author{
Alfredo Ribeiro de Freitas ${ }^{1}$
}

RESUMO - Foram discutidas as propriedades de sete modelos não-lineares, considerando-se o ajuste de curvas de crescimento na produção animal. Os modelos utilizados: Brody, Richards, Von Bertalanffy e duas alternativas de Gompertz e de Logístico foram ajustados, pelo método de Gauss Newton por meio do procedimento NLIN do SAS, a dados peso-idade de oito espécies: camarão-d'água-doce, rãpimenta, coelho, frango, ovino, caprino, suíno e bovino. Considerando-se os critérios como: convergência ou não, coeficiente de determinação e interpretabilidade biológica dos parâmetros, concluiu-se que: a) o modelo Logístico $\mathrm{y}=\mathrm{A} /\left(1+\mathrm{e}^{-\mathrm{kt}}\right)^{\mathrm{m}}$ estimou o peso em todas as espécies animais, enquanto o de Von Bertalanffy apenas não foi adequado para camarão; b) os dois modelos Gompertz foram adequados para camarão, rã, frango, suíno e bovino; c) em cada espécie, pelo menos dois dos sete modelos mostraram-se adequados para estimar o crescimento corporal das espécies animais estudadas, pois os coeficientes de determinação foram superiores a $92,0 \%$.

Palavras-chave: Brody, curvas de crescimento, Gompertz, Logístico, modelos não-lineares, Richards, Von Bertalanffy

\section{Growth Curves in Animal Production}

\begin{abstract}
The properties of seven nonlinear models were discussed concerning its applications in the fitting of growth curves in animal production. The models used: Brody, Richards, Von Bertalanffy and two alternatives of Gompertz and Logistic models, were fitted by Gauss Newton method to weight-age data from eight animal species: freshwater prawn Macrobrachium rosenbergi, pepper frog, rabbit, poultry, sheep, goat, swine and cattle. Considering results of the fitted models such as convergence or not, coefficient of determination and biological interpretation of parameters, it was concluded that: a) the Logisticmethod y = $\mathrm{A} /\left(1+\mathrm{e}^{-\mathrm{kt}}\right)^{\mathrm{m}}$ estimated body weight in all species, while the Von Bertalanffy model was not adequate only for freshwater prawn Macrobrachium rosenbergi; b) both Gompertz models were adequate for freshwater prawn Macrobrachium rosenbergi, pepper frog, poultry, swine and cattle; c) for each specie, at least two nonlinear of the seven models showed adequate to estimate the body weight of the animal species studied, because the coefficients of determination were above $92.0 \%$.
\end{abstract}

Key Words: Brody, Gompertz, growth curves, Logistic, nonlinear models, Richards, Von Bertalanffy

\section{Introdução}

As análises de dados de medidas repetidas são de fundamental importância na produção animal, pois incluem as situações em que as unidades experimentais ou indivíduos, de diferentes subpopulações ou tratamentos (sexo, raça, entre outros), são analisados ao longo de diversas condições de avaliação (tempo, doses etc). Entre essas análises, destacam-se as curvas de crescimento na produção animal, que relacionam os pesos (y) e as idades (t) dos animais, por meio de modelos não-lineares (Davidian e Giltinan, 1996; Paz, 2002).

Entre as várias aplicações das curvas de crescimento na produção animal, destacam-se: a) resumir em três ou quatro parâmetros, as características de crescimento da população, pois alguns parâmetros dos modelos não-lineares utilizados possuem interpretabilidade biológica; b) avaliar o perfil de respostas de tratamentos ao longo tempo; c) estudar as interações de respostas das subpopulações ou tratamentos com o tempo; d) identificar em uma população os animais mais pesados em idades mais jovens; essas informações podem ser obtidas investigando-se o relacionamento entre o parâmetro $\mathrm{k}$ das curvas de crescimento, que expressam a taxa de declínio na taxa de crescimento relativa, e o peso limite do animal ou peso assintótico (Sandland \& Mcgilchrist, 1979; Draper \& Smith, 1980; Davidian \& Giltinam, 1996); e) obter a variância entre e dentro de indivíduos de grande interesse nas avaliações genéticas (Mansour et al., 1991). As aplicações de a) a d) são de interesse geral nos estudos de curvas de crescimento.

Dentro do enfoque de medidas repetidas, é importante considerar duas fontes de variabilidade: variação aleatória entre os animais, que possibilita testar o efeito de tratamentos global e dentro de cada tempo, 
e variação aleatória entre medidas dentro de animal. Nesse caso, tem-se a aplicação das curvas de crescimento, cujo interesse é modelar o padrão de resposta de dados peso-idade ao longo da vida do animal.

O estudo de curvas de crescimento, particularmente em bovinos, é ainda mais atraente, pois os modelos não-lineares são bastante flexíveis para se utilizar com dados peso-idade, pelo fato de considerar características inerentes aos dados de pesagens, como: a) as pesagens são irregulares no tempo, isto é, o intervalo de duas medidas consecutivas quaisquer não é constante; b) possuem estrutura incompleta; c) as avaliações adjacentes são mais estreitamente correlacionadas que as demais; d) a resposta dos indivíduos em função do tempo tem variância crescente.

Tradicionalmente, o ajuste dos modelos não-lineares é feito por meio do procedimento NLIN do SAS (SAS, 1999-2001), utilizando-se o método dos quadrados mínimos ordinários. Entretanto, para solucionar os problemas de heterogeneidade de variâncias e obter estimativas de parâmetros mais eficientes, recomenda-se usar quadrado mínimo generalizado, de modo que as observações com maior variabilidade recebem menos peso na determinação das estimativas de parâmetros. Um dos procedimentos é usar o inverso dos elementos da diagonal da matriz de variânciacovariância da variável dependente, de modo que, quanto maior o peso associado à observação, mais importante ela é para a estimativa de parâmetros (SAS, 1999-2001).

Os objetivos neste trabalho foram: a) ajustar aos dados peso-idade de oito espécies de animais modelos não-lineares tradicionalmente usados na produção animal: Brody, Richards, Von Bertalanffy e duas alternativas de Gompertz e Logístico; b) com base no quadrado médio do resíduo, no coeficiente de determinação e na interpretação biológica dos parâmetros, definir o(s) modelo(s) mais adequado(s) para estimar o crescimento corporal em cada espécie estudada.

\section{Material e Métodos}

Foram utilizados dados peso-idade de oito espécies de animais; os dados foram obtidos de instituições de pesquisas e da literatura, conforme segue: 1 . camarão-d'água-doce (Macrobrachium rosenbergii, de Man, L. 879): os dados utilizados referem-se à media de pesos de camarão-d'àgua-doce cultivados em quatro profundidades (10,20,30 e $40 \mathrm{~cm})$, obtidos do nascimento aos 210 dias de idade, na região Nordeste, no período de maio de 1990 a janeiro de 1991 (Mendes \& Marins, 1995); 2. rã-pimenta: dados de pesos de fêmeas do nascimento aos 10 meses de idade, estimados a partir de modelos ajustados (Agostinho et al., 1991); 3. coelho: dados de machos da raça Nova Zelândia Branco obtidos por pesagens semanais do nascimento aos 70 dias de idade, em Jaboticabal, SP (UNESP); 4. frango: dados de machos da linhagem Pilch obtidos da Embrapa Suinos e Aves; 5. ovinos: dados de 15 pares peso-idade de animais das raças exóticas e mestiças avaliados por animal, do nascimento ao primeiro ano de idade, oriundos da Embrapa Meio-Norte, Teresina, PI; 6. caprino: dados de pesagens trimestrais de animais raça Moxotó do nascimento aos três anos de idade, oriundos da Embrapa Meio-Norte, Teresina, PI; 7. suíno: dados de pesagens de fêmeas Large White ao nascer, aos 21 dias e, semanalmente, dos 60 aos 168 dias de idade, obtidos da Embrapa Suínos e Aves, Concórdia, SC; e 8. bovino: dados de machos da raça Canchim obtidos do nascimento aos 40 meses de idade, obtidos da Embrapa Pecuária Sudeste São Carlos, SP.

Aspectos teóricos dos modelos não-lineares

\section{Modelo de Bertalanffy para estudos metabólicos}

O estudo de curvas de crescimento iniciou-se com o modelo de Bertalanffy para estudos metabólicos (Richards, 1959), dado por:

$$
\mathbf{y}=\left\{\eta / \kappa-\left(\eta / \kappa-\mathbf{y}_{0}^{-m}\right) e^{-(1-m) k t}\right\}^{1 /(1-m)},
$$

em que $\mathbf{y}$ é o peso do animal; $\boldsymbol{\eta}$ e $\boldsymbol{\kappa}$, as constantes de anabolismo e catabolismo, respectivamente; e $\mathbf{y}_{\mathbf{0}}$, o valor de $\mathbf{y}$, quando o tempo $\mathbf{t}$ é igual a zero; para $\mathbf{t} \rightarrow \infty$, o gráfico do modelo converge assintoticamente para

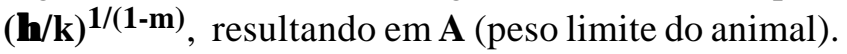
Para $\mathbf{m}=1$, o consumo de oxigênio é proporcional ao peso do animal e $\partial \mathbf{y} / \partial \mathbf{t}=(\boldsymbol{\eta}-\boldsymbol{\kappa}) \mathbf{y}$ e, para $\mathbf{m}=2 / 3$, o metabolismo do animal é proporcional à área da superfície corporal. A taxa de anabolismoé proporcional à m-ésima potência do peso e a taxa de catabolismo, porporcional ao peso em si, de modo que a taxa de crescimento em peso do animal é dada por $\eta \mathbf{y}^{\mathbf{m}}-\kappa \mathbf{y}$.

Modelo de Bertalanffy para estudos de curvas de crescimento

O modelo (1), por proporcionar resultados irreais de $\eta$ e $\kappa$ para situações de $\mathbf{m}>1$, foi reescrito para $\mathbf{y}^{1-\mathbf{m}}=\mathbf{A}^{\mathbf{1 -} \mathbf{m}}-\beta \mathbf{e}^{-\mathbf{K t}}$, em que $\mathrm{A}^{1-\mathbf{m}}=(\boldsymbol{\eta} / \boldsymbol{\kappa})$, 
$\boldsymbol{\beta}=\boldsymbol{\eta} / \mathbf{\kappa}-\mathbf{y}_{\mathbf{0}}^{(1-\mathbf{m})}=\mathbf{A}^{\mathbf{1}-\mathbf{m}}-\mathbf{y}_{\mathbf{0}}^{(\mathbf{1}-\mathbf{m})}, \mathrm{k}=(1-\mathbf{m}) \mathbf{k}$

Posteriormente, foi reparametrizado para:

$$
\mathbf{y}^{1-\mathbf{m}}=\mathbf{A}^{\mathbf{1 - m}}\left(\mathbf{1} \pm \mathbf{b e}^{-\mathbf{K t}}\right)
$$

em que $\mathbf{b}= \pm \boldsymbol{\beta} \mathbf{A}^{\mathbf{1}-\mathbf{m}} ;$ o sinal - quando $\mathbf{m}<\mathbf{1}$ e sinal + para $\mathbf{m}>\mathbf{1}$.

Origem dos modelos Brody, Von Bertalanffy, Logístico, Gompertz e Richards

A partir de (2) e de acordo com o ângulo m (Richards, 1959), estes modelos são obtidos por:

$$
y^{1-m}=A^{1-m}\left(1 \pm b e^{-K t}\right)
$$

Para $\mathbf{0}<\mathbf{m}<\mathbf{1}$, tem-se um modelo intermediário entre Brody e Gompertz; para. $\mathbf{1}<\mathbf{m}<\mathbf{2}$, o formato está situado entre Gompertz e Logístico, ao passo que, para $\mathbf{m}>\mathbf{2}$, resulta em um modelo similar ao Logístico, porém assimétrico, com ponto de inflexão que pode ser alterado para um valor maior que $\mathbf{y}=\mathbf{A} / \mathbf{2}$.

Parâmetros $A, b, K, m$

O parâmetro A é uma estimativa do peso assintótico ou o peso limite, se $\mathbf{t} \rightarrow \infty$; quando o peso adulto do animal não é atingido, A reflete uma estimativa do peso às últimas pesagens. A constante $\mathbf{b}$ não possui interpretação biológica, porém é importante para modelar a curva sigmoidal desde o nascimento $(\mathbf{t}=\mathbf{0})$ até a idade adulta $(\mathbf{t} \rightarrow \infty)$ do animal, resultando nas expressões $\mathbf{A}(\mathbf{1 - b}) \leq \mathbf{y} \leq \mathbf{A} ; \mathbf{A}(\mathbf{1}-\mathbf{b})^{\mathbf{3}} \leq \mathbf{y} \leq \mathbf{A}$; $\mathrm{A} /(\mathbf{1 - b}) \leq \mathrm{y} \leq \mathrm{A} ; \mathrm{Ae}^{-\mathrm{b}} \leq \mathrm{y} \leq \mathrm{A}$ e $\mathrm{A}(\mathbf{1 - b})^{\mathrm{m}} \leq \mathbf{y} \leq \mathrm{A}$, para Brody, Von Bertalanffy, Logístico, Gompertz e Richards, respectivamente; quando $\mathbf{m} \rightarrow \mathbf{1}$, como no caso do Gompertz, tem-se $\mathbf{b}=\left(\mathbf{y}_{\mathbf{0}} / \mathbf{A}\right)^{\mathbf{1 - m}}$.

A constante $\mathrm{k}$ ou índice de maturidade é a razão da taxa de crescimento máxima em relação ao tamanho adulto, a qual expressa a taxa de redução na taxa de crescimento relativa, em $\mathbf{t}^{\mathbf{- 1}}$, em que valores menores indicam taxa de crescimento relativa mais rápida. Segundo Fitzhugh (1976), $\mathbf{k}^{-1}$ é referido como intervalo de maturidade - uma escala de tempo padrão para medir trocas no grau de maturidade.

\section{Taxa de crescimento absoluta instantânea}

A taxa de crescimento absoluta instantânea (TCI) estima o incremento no peso para cada unidade de tempo t; para o modelo geral (3), a TCI é dada por:

$$
\partial \mathbf{y} / \partial \mathrm{t}=\mp K A \quad b e^{-K t}(1-m)^{-1}\left(1 \pm b e^{-K t}\right)^{m /(1-m)}
$$

Substituindo-se os valores de $\mathbf{m}$ de acordo com o item c), em que o sinal + ocorre para $\mathbf{m}<\mathbf{1}$ e o sinal - para $\mathbf{m}>\mathbf{1}$, obtém-se para a TCI: K(A - $\left.\mathbf{y}_{\mathbf{t}}\right), \mathbf{3 b K}$ $\mathbf{y}_{\mathbf{t}} \mathrm{e}^{-K \mathrm{t}}\left(1-\mathrm{e}^{-K \mathrm{t}}\right)^{-1}, K \mathbf{y}_{\mathbf{t}}(\mathrm{A}-\mathrm{y}) / \mathrm{A}, \quad K \mathbf{y}_{\mathbf{t}} \log _{\mathbf{e}}(\mathrm{A} / \mathbf{y}) \mathrm{e}$
$\operatorname{AbKme}^{-K \mathbf{t}}\left(\mathbf{1 - b e} \mathbf{- K t}^{(\mathbf{m}-\mathbf{1})}\right.$, para Brody, Von Bertalanffy, Logístico, Gompertz e Richards, respectivamente; para o Gompertz, o valor da TCI é obtido para $\mathbf{m} \rightarrow \mathbf{1}$, considerando-se a propriedade $\lim _{\mathbf{x} \rightarrow \mathbf{0}}\left(\mathbf{a}^{\mathbf{x}}-1\right)=\log _{\mathbf{e}} \mathbf{a}$.

Taxa de crescimento instantânea relativa (TCIR) $e$ absoluta (TCIA)

A TCIR no tempo $\mathbf{t}$ em relação ao peso do indivíduo nesse particular tempo té obtida do modelo geral (3), de acordo com a variação de $\mathbf{m}$, por:

$$
\begin{gathered}
\mathbf{y}_{\mathbf{t}}^{-1}\left(\partial \mathbf{y}_{\mathbf{t}} / \partial \mathbf{t}\right)=\mp \mathbf{K b e}^{-K t}\left[(\mathbf{1}-\mathbf{m})\left(\mathbf{1} \pm \mathbf{b e}^{-K t}\right)^{-1}\right. \\
(\mp \text { para } \mathbf{m}>\mathbf{1} ; \pm \text { para } \mathbf{m}<\mathbf{1})
\end{gathered}
$$

que resulta nas expressões para a TCIR: $\mathbf{b K e} \mathbf{e}^{-\mathbf{K t}}$ / $\left(1-b e^{-K t}\right), 3 b K e^{-K t} /\left(1-b e^{-K t}\right) ; b K e^{-k t} /\left(1+b e^{-K t}\right)$, $\operatorname{Kylog}_{\mathbf{e}}(\mathrm{A} / \mathrm{y})$ e $-\mathrm{Kbe}^{-\mathrm{Kt}}\left[(\mathbf{1 - m})\left(\mathbf{1}+\mathrm{be}^{-\mathrm{Kt}}\right)^{-\mathbf{1}}\right.$, para Brody, Von Bertalanffy, Logístico, Gompertz e Richards, respectivamente.

A TCIA é vizualizada por meio do gráfico de $\partial \mathbf{y} / \partial \mathbf{t}$, na ordenada e $\mathbf{y}$, na abscissa, a qual aproxima de $\mathbf{A}$ assintoticamente. Uma vez que b é aproximadamente 1, a forma do gráfico depende dos parâmetros $\mathbf{A}, \mathbf{m}$ e $\mathbf{K}$; a área da curva é dada porque é igual a $A^{\mathbf{2}} \mathbf{K} /(\mathbf{2 m}+\mathbf{2})$ e a altura média, por $\mathbf{A K} /(\mathbf{2 m}+\mathbf{2})$, que representa a taxa de crescimento média dos animais dentro da população. A taxa de crescimento relativa média de uma população no ponto de inflexão é representada por: $\mathbf{k} / \mathbf{m}$

\section{Ponto de inflexão (PI)}

É o ponto em que a TCI passa de crescente para decrescente. É dado pela equação $\mathbf{y}_{\mathbf{t}} / \mathbf{A}=\mathbf{m}^{\mathbf{1} /(\mathbf{1}-\mathbf{m})}$, correspondendo a $\mathbf{y}_{\mathfrak{t}} / \mathbf{A}=\mathbf{8 / 2 7}, \mathbf{y}_{\mathfrak{t}} / \mathbf{A}=\mathbf{1} / \mathbf{2}, \mathbf{y}_{\mathfrak{t}} / \mathbf{A = 1 / e}$ e $\mathbf{y}_{\mathfrak{t}}$ / $\mathbf{A}=\mathbf{m}^{\mathbf{1} /(\mathbf{1}-\mathbf{m})}$, para Von Bertalanffy, Logístico, Gompertz e Richards, respectivamente. Observa-se que a constante $\mathbf{m}$ determina a proporção do peso final (A) no qual o PI ocorre. No caso particular do modelo Brody, que não possui PI (indefinido para $\mathrm{m}=0$ ), é comum utilizar este modelo para descrever o crescimento pós-natal ou fase de auto-inibição do crescimento.

\section{Taxa de maturidade absoluta}

É a razão de $\partial \mathbf{y}_{\mathbf{t}} / \partial \mathbf{t}$ em relação ao peso assintótico $\mathbf{A}$, ou seja, $\mathbf{A}^{\mathbf{- 1}} \partial \mathbf{y}_{\mathfrak{t}} / \partial \mathbf{t}$. O gráfico $\mathbf{A}^{\mathbf{- 1}} \partial \mathbf{y} / . \partial \mathbf{t}$ versus $\mathbf{y}_{\mathbf{t}} / \mathbf{A}$ representa a taxa de troca em peso do tamanho proporcional em relação ao tamanho global, variando na escala de 0 a 1 . A área delimitada pela curva é igual a $\mathbf{k} /(\mathbf{2 m}+\mathbf{2})$ e possibilita aplicações importantes. Quando se faz o ajuste de uma curva a um conjunto de animais, tem-se $\mathbf{m}$ constante e a área é dependente 
apenas da constante K. Nesse caso, por meio da associação de k e o peso limite do animal, é possível identificar na população os animais mais pesados e mais jovens. Da mesma forma, quando diferentes curvas são ajustadas ao um conjunto de animais, pode-se estabelecer uma equivalência entre os k's e comparar estas curvas variando-se apenas o valor de $\mathbf{m}$. Por exemplo, para k/2, k/4, k/6 e k/(10/3), os modelos Brody, Gompertz, Logístico e Bertalanffy, respectivamente, podem ser comparados variando-se apenas o valor de $\mathrm{m}$.
Taxa de crescimento no intervalo $t_{i}$ e $t_{j}(i<j)$

Para duas avaliações quaisquer i e j no animal, a taxa de crescimento média é dada por $1 /\left(\mathrm{t}_{\mathrm{j}}-\mathrm{t}_{\mathrm{i}}\right) \int_{t_{i}}^{t_{j}}$ $(\partial y / \partial t) \partial t=\left(y_{j}-y_{i}\right) /\left(t_{j}-t_{i}\right)$, enquanto a taxa de maturidade absoluta média, por $\mathrm{A}^{-1} /\left(\mathrm{t}_{\mathrm{j}}-\mathrm{t}_{\mathrm{i}}\right) \int_{t_{i}}^{t_{j}} \partial \mathrm{y} /$ $\partial t \partial t=A^{-1}\left(y_{j}-y_{i}\right) /\left(t_{j}-t_{i}\right)$.

Tabela 1 - Propriedades dos modelos não-lineares (y): Brody (1), Gompertz (2a, 2b), Logístico (3a, 3b), Richards (4) e Von Bertalanffy (5). Taxa de crescimento instantânea ( $\partial \mathrm{y} / \partial \mathrm{t})$, taxa de maturidade absoluta $\left(\mathrm{A}^{-1}[\partial \mathrm{y} / \partial \mathrm{t}]\right)$, taxa de crescimento instantânea relativa $((\partial y / \partial t) / y)$ e ponto de inflexão $\left(y_{i} ; t_{i}\right)$

Table 1 - Properties of nonlinear models: Brody (1), Gompertz (2a, 2b), Logistic (3a, 3b), Richards (4) and Von Bertalanffy (5). Instantaneous growth rate $(\partial y / \partial t)$, absolute maturity rate $\left(A^{-1}[\partial y / \partial t]\right)$, relative instantaneous growth rate $\left.(\partial y / \partial t) / y\right)$ and infletion point $\left(y_{i} ; t_{i}\right)$

\begin{tabular}{|c|c|c|c|c|}
\hline Modelo: $\quad \mathrm{y}=$ & $\partial \mathbf{y} / \partial \mathbf{t}$ & $\mathrm{A}^{-1}(\partial \mathbf{y} / \partial \mathbf{t})$ & $(\partial \mathbf{y} / \partial \mathbf{t}) / \mathrm{y}$ & $\left(\mathrm{y}_{\mathrm{i}} ; \mathrm{t}_{\mathrm{i}}\right)$ \\
\hline $\begin{array}{l}\text { 1.A(1-bC } 1) \\
\partial \mathrm{y} / \partial \mathrm{A}=\left(1-\mathrm{bC}_{1}\right) \\
\partial \mathrm{y} / \partial \mathrm{b}=-\mathrm{AC}_{1} \\
\partial \mathrm{y} / \partial \mathrm{K}=\mathrm{AbtC}_{1}\end{array}$ & $\mathrm{AbkC1}$ & bkC1 & $\mathrm{AbkC} 1 / \mathrm{y}$ & não possui \\
\hline $\begin{array}{l}\text { 2a. yo.exp }\left[(\mathbf{L} / \mathbf{K})\left(\mathbf{1}-\mathbf{C}_{\mathbf{1}}\right)\right] \\
\partial \mathrm{y} / \partial \mathrm{y}_{\mathrm{o}}=\exp \left\{(\mathrm{L} / \mathrm{K})\left(1-\mathrm{C}_{1}\right)\right\} \\
\partial \mathrm{y} / \partial \mathrm{K}=\mathrm{yLtC} 1 / \mathrm{K} \\
\partial \mathrm{y} / \partial \mathrm{L}=\mathrm{y}\left(1-\mathrm{C}_{1}\right) / \mathrm{K}\end{array}$ & $\mathrm{LyC}_{1}$ & $\mathrm{LyC}_{1} / \mathrm{A}$ & $\mathrm{LC}_{1}$ & $0,368 \mathrm{~A} ; \log \mathrm{e}(\mathrm{L} / \mathrm{K}) / \mathrm{K}$ \\
\hline $\begin{array}{l}\text { 2b. } \operatorname{Aexp}\left(-\mathbf{b C}_{\mathbf{1}}\right) \\
\partial \mathrm{y} / \partial \mathrm{A}=\exp \left(-\mathrm{bC}_{1}\right) \\
\partial \mathrm{y} / \partial \mathrm{b}=-\mathrm{yC}_{1} \\
\partial \mathrm{y} / \partial \mathrm{K}=\mathrm{ybtC}_{1}\end{array}$ & bkyC1 & $\mathrm{kyC} 1 / \mathrm{A}$ & bkyC1 & $\mathrm{A} / \mathrm{e} ;(\log \mathrm{b}) / \mathrm{k}$ \\
\hline $\begin{array}{l}\text { 3a. } \mathbf{A} /\left(\mathbf{1}+\mathbf{C}_{\mathbf{1}}\right)^{\mathrm{m}} \\
\partial \mathrm{y} / \partial \mathrm{A}=1 /\left(1+\mathrm{C}_{1}\right)^{\mathrm{m}} \\
\partial \mathrm{y} / \partial \mathrm{k}=\mathrm{ymtC} /\left(1+\mathrm{C}_{1}\right) \\
\partial \mathrm{y} / \partial \mathrm{m}=-\mathrm{y} \ln \left(1+\mathrm{C}_{1}\right)\end{array}$ & $\mathrm{KmyC}_{1} /\left(1+\mathrm{C}_{1}\right)$ & $\mathrm{KmC}_{1}\left(1+\mathrm{C}_{1}\right)^{-\mathrm{m}-1}$ & $\mathrm{KmC}_{1} /\left(1+\mathrm{C}_{1}\right)$ & $\mathrm{A}[\mathrm{m} /(\mathrm{m}+1)]^{\mathrm{m}} ; \log _{\mathrm{e}} \mathrm{m} / \mathrm{K}$ \\
\hline $\begin{array}{l}\text { 3b. } \mathbf{A} /\left(\mathbf{1}+\mathbf{b C}_{\mathbf{1}}\right) \\
\partial \mathrm{y} / \partial \mathrm{A}=1 /\left(1+\mathrm{bC}_{1}\right) \\
\partial \mathrm{y} / \partial \mathrm{b}=-\mathrm{yC}_{1} /\left(1+\mathrm{bC}_{1}\right) \\
\partial \mathrm{y} / \partial \mathrm{K}=\mathrm{ybt} /\left(1+\mathrm{bC}_{1}\right) \mathrm{C}_{1}\end{array}$ & $\mathrm{ybk} /\left(1+\mathrm{bC}_{1}\right) \mathrm{C}_{1}$ & $\mathrm{bkC}_{1} /\left(1+\mathrm{bC}_{1}\right)^{2}$ & $\mathrm{bk} /\left(1+\mathrm{bC}_{1}\right) \mathrm{C}_{1}$ & $\mathrm{~A} / 2 ;(\operatorname{lnb}) / \mathrm{K}$ \\
\hline $\begin{array}{l}\text { 4. } \mathbf{A}\left(1-\mathrm{bC}_{1}\right)^{\mathbf{m}} \\
\partial \mathrm{y} / \partial \mathrm{A}=\left(1-\mathrm{bC}_{1}\right)^{\mathrm{m}} \\
\partial \mathrm{y} / \partial \mathrm{b}=-\mathrm{ymC}_{1} /\left(1-\mathrm{bC}_{1}\right) \\
\partial \mathrm{y} / \partial \mathrm{K}=\mathrm{ymbt} \mathrm{C}_{1} /\left(1-\mathrm{bC} \mathrm{C}_{1}\right) \\
\partial \mathrm{y} / \partial \mathrm{m}=\mathrm{y} \ln \left(1-\mathrm{bC}_{1}\right)\end{array}$ & $\operatorname{ymbkC}_{1} /\left(1-b_{1}\right)$ & $\mathrm{mbkC}_{1}\left(1-\mathrm{bC}_{1}\right)^{(\mathrm{m}-1)}$ & $\mathrm{mbkC}_{1} /\left(1-\mathrm{bC}_{1}\right)$ & $\mathrm{Am}^{1 /(1-\mathrm{m})} ; \ln \left(\mathrm{b}(1-\mathrm{m})^{-1}\right) / \mathrm{K}$ \\
\hline $\begin{array}{l}\text { 5. } \mathbf{A}\left(\mathbf{1}-\mathbf{b C}_{\mathbf{1}}\right)^{\mathbf{3}} \\
\partial \mathrm{y} / \partial \mathrm{A}=\left(1-\mathrm{bC}_{1}\right)^{3} \\
\partial \mathrm{y} / \partial \mathrm{B}=-3 \mathrm{AC} \mathrm{C}_{1}\left(1-\mathrm{bC}_{1}\right)^{2} \\
\partial \mathrm{y} / \partial \mathrm{K}=3^{\mathrm{A}} \mathrm{BT}\left(1-\mathrm{bC}_{1}\right)^{2} \mathrm{C}_{1} \\
\mathrm{C}_{1}=\exp (-\mathrm{kt})\end{array}$ & $3 \mathrm{AbkC1}\left(1-\mathrm{bC}_{1}\right)^{2}$ & $3 \mathrm{bkC}\left(1-\mathrm{bC}_{1}\right)^{2}$ & $3 \mathrm{ybKC}_{1} /\left(1-\mathrm{bC}_{1}\right)$ & $8 \mathrm{~A} / 27 ; \operatorname{loge}(3 \mathrm{~b}) / \mathrm{K}$ \\
\hline
\end{tabular}




\section{Modelos analisados}

Constam na Tabela 1 os modelos não-lineares usados no presente trabalho, assim como algumas propriedades associadas aos mesmos, como taxa de crescimento absoluta, taxas de crescimento instantânea relativas e ponto de inflexão. Além dos cinco modelos do item 1 , foram considerados dois modelos alternativos: o Gompertz, dado por: $\mathrm{y}=\mathrm{y}_{\mathrm{o}} \cdot \exp [(\mathrm{L} / \mathrm{K})$ $\left.\left(1-\mathrm{e}^{-\mathrm{kt}}\right)\right]$ (Freitas e Costa, 1983), em que o termo $\mathrm{y}_{\mathrm{o}} \mathrm{e}^{\mathrm{L} / \mathrm{K}}$ e L/K, equivale, respectivamente, ao parâmetro A e b, e o Logístico: $\mathrm{y}=\mathrm{A} /\left(1+\mathrm{e}^{-\mathrm{kt}}\right)^{\mathrm{m}}$, descrito por Fitzhugh (1976). O parâmetro L não tem significado biológico, porém, juntamente com o $\mathrm{k}$ compõem o $\mathrm{b}$, que tem a função de modelar a curva sigmoidal.

A escolha dos modelos mais adequados para cada espécie animal, foram feitas considerando-se: a) convergência ou não (o processo iterativo converge na j-ésima iteração quando $\left.\mathrm{SQR}_{\mathrm{j}-1}-\mathrm{SQR}_{\mathrm{j}}\right) /\left(\mathrm{SQR}_{\mathrm{j}}+10^{-6}\right)$ $<10^{-8}$; b) coeficiente de determinação dado por 1- (SQR/SQT), em que SQR e SQT é a soma de quadrados do resíduo e total corrigida, respectivamente e c) interpretabilidade biológica dos parâmetros. O ajuste dos modelos aos dados foi feito por meio do procedimento NLIN (SAS) e do método iterativo de Gauss-Newton.

\section{Resultados e Discussão}

As estimativas dos parâmetros dos modelos nãolineares, por espécie estão na Tabela 2. Para os dados de todas as espécies, foram ajustados os modelos desta tabela, sendo a convergência ou não do modelo o critério inicial de ajuste. Para todas as situações em que o ajuste foi obtido, os coeficientes de determinação foram superiores a 92,0\%, mostrando que o crescimento dos animais foi estimado adequadamente nessas situações. O número de modelos que se mostraram adequados por espécie foram 3, 5, 2, 5, 3, 4, 4, 5, para camarão-d'água-doce, rã-pimenta, coelho, frango, ovino, caprino, suíno e bovino, respectivamente, ou seja, pelo menos dois modelos mostraram-se adequados para estimar o crescimento corporal dos dados peso-idade das espécies consideradas. Os modelos mais versáteis foram o Logístico $\mathrm{y}=\mathrm{A} /\left(1+\mathrm{e}^{-\mathrm{kt}}\right)^{\mathrm{m}}$ que estimou o peso em todas as espécies, seguido do Von Bertalanffy, que não ajustou dados de camarão, e os de Gompertz, que estimaram o crescimento para camarão, rã, frango, suíno e bovino. Uma vez que a qualidade de ajuste de um modelo a uma espécie animal, entre outros fatores, é dependente do número de pares peso-idade avaliados (sexo, raça, manejo, idade do animal) em que o último peso é obtido, a expectativa é que as estimativas de parâmetros obtidas neste trabalho sejam usadas como valores iniciais em situações específicas do usuário.

A Figura 1 ilustra o gráfico de uma curva de crescimento por espécie, selecionada de modo a ilustrar a maior diversidade de modelos possível. Das oito curvas ajustadas, a única que não apresentou o aspecto sigmoidal é a de caprino.

Mendes \& Marins (1995) estimaram o crescimento em peso e comprimento e a taxa de sobrevivência de camarões Macrobrachium rosenbergii, cultivados a 10, 20, 30 e $40 \mathrm{~cm}$ da coluna de água, do nascimento até 210 dias de idade pelo modelo de Von Bertalanffy. Os autores verificaram que os pesos máximos assintóticos foram de 11,4; 13,7; 14,7 e 23,8 $\mathrm{g}$, respectivamente, para os cultivos de 10, 20, $30 \mathrm{e}$ $40 \mathrm{~cm}$ da coluna de água, porém, a partir de 180 dias de cultivo, os valores observados foram maiores que os estimados; aos 210 dias, as médias de pesos $(\mathrm{g})$ foram 11,$96 ; 11,81 ; 17,92$ e 20,16, para $10,20,30 \mathrm{e}$ $40 \mathrm{~cm}$ da coluna de água. No presente trabalho, quando se utilizou para o ajuste os mesmos dados, porém, considerando-se a média de pesos de camarão cultivados nas quatro profundidades, verificou-se que ambos os modelos Gompertz e Logístico $\mathrm{y}=\mathrm{A} /(1+$ $\left.\mathrm{e}^{-\mathrm{kt}}\right)^{\mathrm{m}}$ (Tabela 2) foram adequados, não havendo distinção entre eles. No entanto, os três modelos superestimaram os pesos iniciais e os últimos pesos.

Com exceção do Brody e do logístico y = $\mathrm{A} /\left(1+\mathrm{be}^{-\mathrm{kt}}\right)$, todos os modelos estudados (Tabela 2) estimaram adequadamente o crescimento de rãs até os 10 meses de idade. Para os mesmos dados, Agostinho et al., (1991), utilizando apenas o modelo de Von Bertalanffy, concluíram que o mesmo superestimou o peso inicial. Porém, para as demais idades, houve correspondência entre os pesos observados e os estimados. Observou-se boa concordância entre o valor assintótico estimado por aqueles autores $(329,1 \mathrm{~g})$ com o estimado no presente trabalho $(322,1 \mathrm{~g})$.

Das espécies estudadas, o ajuste de curvas de crescimento para coelho (Tabela 2) foi o mais difícil, pois apenas o modelo Logístico $\mathrm{y}=\mathrm{A} /\left(1+\mathrm{C}_{1}\right)^{\mathrm{m}}$ e Von Bertalanffy foram adequados para estimar o crescimento. Ambos os modelos subestimaram os pesos iniciais e finais. Entretanto, na fase inicial, o Bertalanffy foi inferior ao Logístico; nas fases intermediárias e finais, os dois apresentaram comportamento semelhantes. 
Tabela 2 - Estimativas de parâmetros de modelos não-lineares em oito espécies animais: Brody (1), Gompertz (2a, 2b), Logístico(3a, 3b), 4: Richards e 5: Von Bertalanffy e espécie. A parte sombreada indica a espécie que o modelo não se ajustou

Table 2 - Parameter estimates of nonlinear model in eight animal species: Brody(1), Gompertz(2a,2b), Logistic(3a,3b), Richards (4) and Von Bertalanffy (5) and species. The dashed area indicates that the model did not fit*

\begin{tabular}{l}
$\mathbf{y}=$ \\
\hline 1. A(1-bC \\
$\mathbf{A}, \mathbf{k g}$ \\
$\mathbf{b}, \mathbf{k g} / \mathbf{k g}$ \\
$\mathbf{K}, \mathbf{t}^{-1}$ \\
$\mathbf{R}^{\mathbf{2}}$
\end{tabular}

2a. yo,exp[(L/K)(1- $\left.\left.\mathrm{C}_{1}\right)\right]$

$\begin{array}{lll}\mathbf{\mathbf { y } _ { \mathbf { o } }}, \mathbf{k g} & 0,7528 & 0,0121 \\ \mathbf{L}, \mathbf{g} / \mathbf{g} & 0,0184 & 0,6243 \\ \mathbf{K}, \mathbf{t}^{-1} & 0,0026 & 0,2069 \\ \mathbf{R}^{\mathbf{2}} & 0,9799 & 0,9999 \\ \left.\text { 2b. Aexp(-bC }{ }_{1}\right) & & \end{array}$

\begin{tabular}{cccc} 
Camarão & Rã & Coelho & Frango \\
Freshwater & Pepperfrog & Rabbit & Poultry \\
\hline
\end{tabular}

$\begin{array}{cc}\text { Caprino } & \text { Ovino } \\ \text { Goat } & \text { Sheep }\end{array}$

Suíno
Swine $\quad \begin{gathered}\text { Cattle } \\ \text { Covino }\end{gathered}$

$\mathrm{A}, \mathbf{k g}$

$\mathbf{b}, \mathbf{k g} / \mathbf{k g}$
$\mathbf{K}^{2} \mathbf{t}^{-1}$

0,9562

0,2477

$\begin{array}{ll}7,1469 & 3,0138 \\ 0,0026 & 0,2066\end{array}$

$0,9799 \quad 0,9999$

$\begin{array}{cc}\mathbf{5 6 , 9 9 6 8} & 39,8745 \\ \mathbf{0 , 8 9 2 3} & 0,9260 \\ \mathbf{0 , 0 5 0 8} & 0,0040 \\ 0,9391 & 0,9372\end{array}$

1751,9000

0,9734

0,0103

0,9244

3a. $\mathrm{A} /\left(1+\mathrm{C}_{1}\right)^{\mathrm{m}}$

$\mathbf{A}, \mathbf{k g}$
$\mathbf{K}, \mathbf{t}^{-1}$
$\mathbf{M}, \mathbf{k g} / \mathbf{k g}$
$\mathbf{R}^{\mathbf{2}}$
3b. A/(1+bC 1$)$

A, kg

$\mathrm{b}, \mathrm{kg} / \mathrm{kg}$

$\mathbf{K}, \mathbf{t}^{-1}$

$\mathbf{R}^{2}$

$\mathbf{0 , 4 5 6 2} 0,2213$

$\mathbf{0 , 0 0 3 8} 0,2651$

4,0810

$\mathbf{9 , 1 7 2 0}$

0,9999

0,0265

0,1669

0,0341

0,9444

5,0148

4,8905

$\mathbf{0 , 0 3 4 1}$

0,9444

\section{$\mathbf{2 , 0 2 7 0} \quad 4,6257$}

0,0459

$\mathbf{5 , 6 6 3 2}$

0,9412

0,0404

6,4917

0,9999

3,5597

33,9103

0,0776

0,9341

\section{$\mathbf{1 , 7 4 2 1} 72,7672$ \\ $\mathbf{0 , 0 6 0 0} 0,1343$ \\ $\mathbf{0 , 0 1 3 2} 0,0566$ \\ $0,9500 \quad 0,9232$}

$164,1111 \quad 780,5950$

$0,0600 \quad 2,3728$

$0,0132 \quad 0,0566$

$0,9500 \quad 0,9232$

$\begin{array}{cccc}49,6414 & 31,7670 & 149,3101 & 731,0000 \\ 0,1164 & 0,0114 & 0,0169 & 0,0713 \\ 2,4913 & 2,8403 & 6,4020 & 3,2265 \\ 0,9332 & 0,9390 & 0,9320 & 0,9357\end{array}$

4. $\mathrm{A}\left(1-\mathrm{bC}_{1}\right)^{\mathrm{m}}$

A, $\mathrm{kg}$

$\mathrm{b}, \mathrm{kg} / \mathrm{kg}$

0,3221

0,6560

$\mathrm{K}, \mathrm{t}^{-1}$

0,1295

$\mathrm{m}, \mathrm{kg} / \mathrm{kg}$

3,1756

$\mathbf{R}^{2}$

0,9999

5. $\mathrm{A}\left(1-\mathrm{bC}_{1}\right)^{3}$

\begin{tabular}{lcccccccc}
$\mathbf{A}, \mathbf{k g}$ & & 0,3294 & $\mathbf{2 , 6 2 2 3}$ & 7,5453 & 51,9850 & $\mathbf{3 4 , 0 1 7 2}$ & 329,0520 & $\mathbf{8 7 2 , 9 0 0 0}$ \\
$\mathbf{b , \mathbf { k g } / \mathbf { k g }}$ & & 0,6800 & $\mathbf{0 , 8 2 9 8}$ & 0,8872 & 0,4733 & $\mathbf{0 , 5 1 8 3}$ & 0,8387 & $\mathbf{0 , 5 7 7 7 7}$ \\
$\mathbf{K}, \mathbf{t}^{-1}$ & & 0,1249 & $\mathbf{0 , 0 2 4 7}$ & 0,0184 & 0,0823 & $\mathbf{0 , 0 0 7 6}$ & 0,0056 & $\mathbf{0 , 0 4 1 3}$ \\
$\mathbf{R}^{\mathbf{2}}$ & 0,9999 & 0,9402 & 0,9999 & 0,9370 & 0,9393 & 0,9244 & 0,9238 & \\
\hline
\end{tabular}

$\mathrm{C}_{1}=\exp (-\mathrm{kt})$.

*As estimativas em negrito foram usadas na elaboração da Figura 1.

* The estimates in bold were used in the construction of Figure 1.

Para frangos, as duas formas de Gompertz, Logístico e ainda Von Bertalanffy apresentaram bom ajuste (Tabela 2). Quanto aos pesos iniciais, ambos os modelos Gompertz e Von Bertalanffy subestimaram os pesos iniciais até 12 dias de idade, enquanto os Logísticos superestimaram até oito dias. Para as idades intermediárias, os cinco modelos proporcionaram bom ajuste, mas todos subestimaram a partir de
65 dias. Freitas et al. (1984) compararam entre si regressões polinomiais e os modelos Gompertz, Logístico, Richards e Von Bertalanffy, para ajustar dados peso-idade de frangos e frangas do nascimento até 68 dias de idade, e concluíram que o modelo Gompertz y $=\mathrm{y}_{\mathrm{O}}$. $\exp \left[(\mathrm{L} / \mathrm{K})\left(1-\mathrm{e}^{-\mathrm{kt}}\right)\right]$ e o Logístico $\mathrm{y}=$ $\mathrm{A} /\left(1+\mathrm{e}^{-\mathrm{kt}}\right)^{\mathrm{m}}$ proporcionaram os resultados mais adequados para o crescimento. 

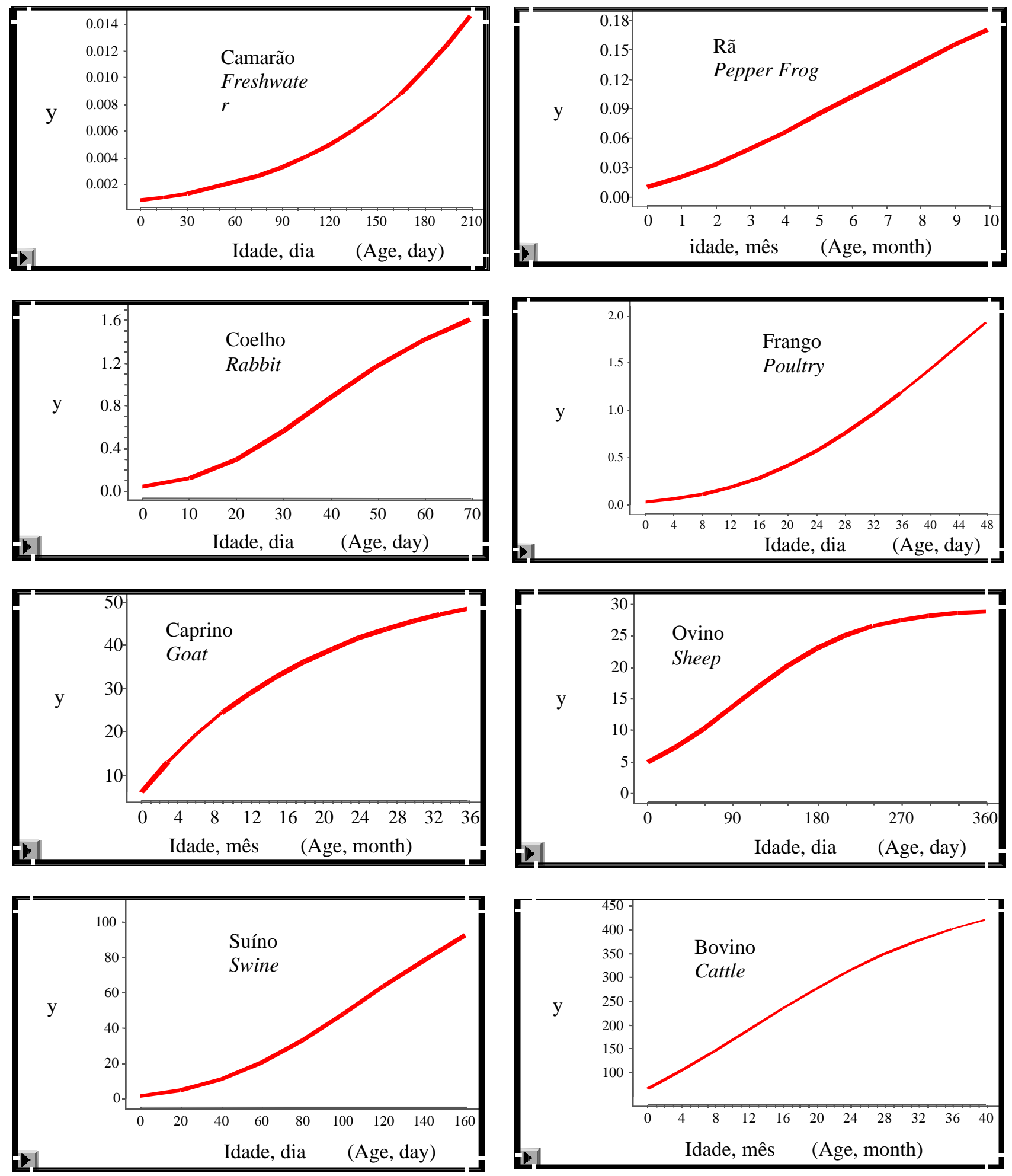

Figura 1 - Estimativas de pesos, em kg, por modelo e espécie animal: Logística (camarão), Bertalanffy (rã), Logística (coelho), Gompertz (frango), Brody (caprino), Bertalanffy (ovino), suíno (Gompertz) e Gompertz (bovino).

Figure 1 - Weight estimates, in $\mathrm{kg}$, according to the model and specie: Logistic ( freshwater), Bertalanffy (Pepper frog) Logistic (rabbit), Gompertz (poultry), Brody (goat), Bertalanffy (sheep), Gompertz (swine) and cattle (Gompertz). 
Três modelos (Brody, o Logístico y $=\mathrm{A} /\left(1+\mathrm{e}^{-\mathrm{kt}}\right)^{\mathrm{m}}$ e o Von Bertalanffy) foram adequados (Tabela 2) para estimar o crescimento de caprinos, em todas as idades, sendo a única exceção o peso ao nascimento, pois os três modelos o superestimaram em magnitude de $10 \%$. Observa-se que o gráfico da Figura 1 não apresenta o aspecto sigmoidal, pois foi ajustado o modelo Brody, que não possui o ponto de inflexão, conforme descrito no item Ponto de inflexão (PI) de Material e Métodos. Cardoso Monteiro et al. (1999) aplicaram os modelos de Brody, Richards, Gompertz, Von Bertalanffy e o Logístico, para dados de bodes Serranos Transmontanos. Segundo esses autores, o modelo de Richards proporcionou o melhor ajuste dos dados $\left(\mathrm{R}^{2}=0,97\right)$, porém, os modelos Brody e Von Bertalanffy ajustaram razoavelmente os dados. Todos os modelos apresentaram ajuste pobre para as fases iniciais de crescimento.

O ajuste para ovinos foi semelhante ao de caprinos, exceto que para esta espécie o modelo Logístico $\mathrm{y}=\mathrm{A} /\left(1+\mathrm{be}^{-\mathrm{kt}}\right)$ foi adequado. A curva exibida na Figura 1, ajustada com o modelo Von Bertalanffy, é do tipo sigmóide e mostra estabilização no crescimento, ou seja, para esta curva o parâmetro A fornece indicação do peso à maturidade. Santos et al. (2001), estudando o crescimento de ovinos da raça Santa Inês desde o nascimento, mostraram que, em condições ambientais adequadas, a curva descrita é do tipo sigmóide, havendo aceleração da sua velocidade até que a puberdade seja atingida, diminuindo gradativamente até a maturidade.

Para a espécie suína, os estudos de curvas de crescimento são mais abundantes que para as demais espécies citadas e os resultados são semelhantes. No presente trabalho (Tabela 2), os modelos adequados foram o de Gompertz $y=y_{0} \cdot \exp \left[(L / K)\left(1-e^{-k t}\right)\right]$, o Logístico $\mathrm{y}=\mathrm{A} /\left(1+\mathrm{e}^{-\mathrm{kt}}\right)^{\mathrm{m}}$ e Von Bertalanffy. Freitas \& Costa (1983) e Rodrigues et al. (1992), estudando as diferentes funções (Bertalanffy, Logístico, Gompertz e Richards) para estimarem curvas de crescimento de suínos, do nascimento ao abate, concluíram que os modelos tiveram bom ajuste dos dados, com altos valores de $\mathrm{R}^{2}$. Dutra Jr. et al. (2001) citam também que o tecido muscular, adiposo e ósseo de suíno também são estimados com boa acurácia por curvas de crescimento. O fato de o modelo Brody não ter se ajustado nesta pesquisa corrobora os resultados encontrados na literatura. Segundo Alves (1986), citado por Dutra Jr. et al.(2001), este foi o de pior ajuste.
No ajuste de dados para bovinos, pelo fato de se dispor somente de dados de pesos mensais, do nascimento aos 40 meses de idade de bovinos machos Canchim, esta espécie foi escolhida para ilustrar algumas das propriedades descritas anteriormente. Entre os modelos estudados para esta espécie (Tabela 2), observa-se que apenas o Logístico $\mathrm{y}=\mathrm{A} /\left(1+\mathrm{be}^{-\mathrm{kt}}\right)$ e Richards não se ajustaram aos dados; o gráfico da Figura 1 ilustra o ajuste com o modelo de Von Bertalanffy. As estimativas de pesos $(\mathrm{kg})$ e a taxa de crescimento no instante $\mathrm{t}$ (TCI), em kg/mês, são apresentadas nas Figura 2 e 3, respectivamente, em que os índices de 1 a 4 , indicam os modelos Brody, Gompertz y =Aexp(-be $\left.{ }^{-k t}\right)$, Logístico $\mathrm{y}=\mathrm{A} /\left(1+\mathrm{e}^{-\mathrm{kt}}\right)^{\mathrm{m}}$ e Von Bertalanffy. Observando-se o comportamento dos modelos quanto a estimativas de pesos (Figura 2), verifica-se que eles proporcionaram resultados semelhantes, porém, há diferença entre os mesmos na fase inicial e às últimas pesagens. Na fase inicial, o mais indicado foi o Brody, enquanto os demais tenderam a superestimar os pesos. Entretanto, considerando-se todos os pares peso-idade, o Logístico $\mathrm{y}=\mathrm{A} /\left(1+\mathrm{e}^{-\mathrm{kt}}\right)^{\mathrm{m}}$, seguido de Von Bertalanffy, foi o mais indicado.

As estimativas de pesos $(\mathrm{kg})$ ao nascimento obtidas a partir destes modelos foram 46,6; 72,7; 89,4 e

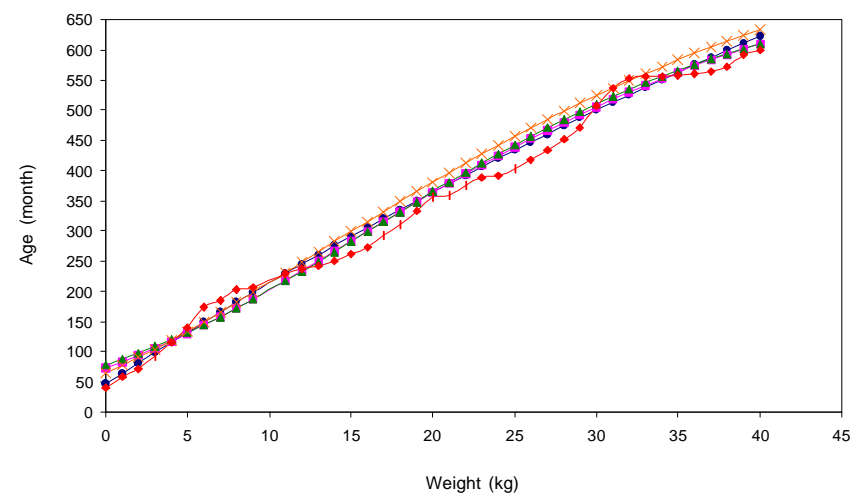

Figura 2 - Médias de pesos observados, em kg (Weight, $\mathrm{kg}$ ) em função da idade, em mês (Age, month) de bovinos machos Canchim, obtidas dos modelos: 1) Brody: $\mathrm{y}_{1}=\mathrm{A}\left(1-\mathrm{be}^{-\mathrm{kt}}\right) ;$ 2) Gompertz: $\left.\mathrm{y}_{2}=A \exp \left(-\mathrm{be}^{-\mathrm{kt}}\right) ; 4\right)$ Logístico: $\mathrm{y}_{3}=\mathrm{A} /(1+\exp (-$ $k \mathrm{t}))^{\mathrm{m}}$ e 5) Von Bertalanffy: $\mathrm{y}_{4}=\mathrm{A}\left(1-\mathrm{be}^{-\mathrm{kt}}\right)^{3}$, cujos índices são os mesmos dos gráficos.

Figure 2 - Average observed weights, in $\mathrm{kg}$, as a function of age, in month, from male Canchim cattle obtained from the models: Brody: $y_{1}=A\left(1-b e^{-k t}\right)$; Gompertz: $y_{2}$ $=A \exp \left(-b e^{-k t}\right)$; Logistic: $y_{3}=A /(1+\exp (-k t))^{m}$ and Von Bertalanffy: $y_{4}=A\left(1-b e^{-k t}\right)^{3}$, whose indices are the same of the plots. 
78,0, para Brody, Gompertz, Logístico e Von Bertalanffy, respectivamente; todas superiores ao valor observado $(40,2 \mathrm{~kg})$. Parece haver tendência dos modelos ajustados superestimarem os pesos iniciais, pois, em estudos de crescimento de fêmeas bovinas de várias raças, Brown et al. (1976) verificaram que os modelos Gompertz, Logístico e o Von Bertalanffy superestimaram os pesos iniciais.

Uma análise mais detalhada da Figura 2 possibilita verificar que os modelos superestimaram os pesos até os dois meses, dos 23 aos 28 e dos 30 aos 34 meses de idade e subestimaram dos 6 aos 12 meses. Aos 40 meses de idade, os pesos estimados, em $\mathrm{kg}$, foram 622,$4 ; 610,0 ; 609,9$ e 613,9 para Brody, Gompertz (duas alternativas), Logístico $\mathrm{y}=\mathrm{y}=\mathrm{A} /\left(1+\mathrm{e}^{-\mathrm{kt}}\right)^{\mathrm{m}}$ e Von Bertalanffy, respectivamente, os quais superestimaram o valor observado $(600,2 \mathrm{~kg})$. Entretanto, pode-se observar (Tabela 2), que estes modelos foram adequados para estimar o crescimento corporal de animais Canchim até os 40 meses de idade.

Quanto à taxa de crescimento no instante t -TCI, em $\mathrm{kg} / \mathrm{mês}$ (Figura 3), verifica-se que do nascimento até o $40^{0}$ mês, os valores variaram de 17,5 a $11,6 \mathrm{~kg}$; 9,8 a $8,5 \mathrm{~kg} ; 9,0$ a $7,6 \mathrm{~kg}$; e 11,1 a 9,5 para $\mathrm{y}_{1}$ a $\mathrm{y}_{4}$, respectivamente. Excetuando-se o modelo Brody $\left(\mathrm{y}_{1}\right)$, que não apresenta ponto de inflexão (PI), os valores

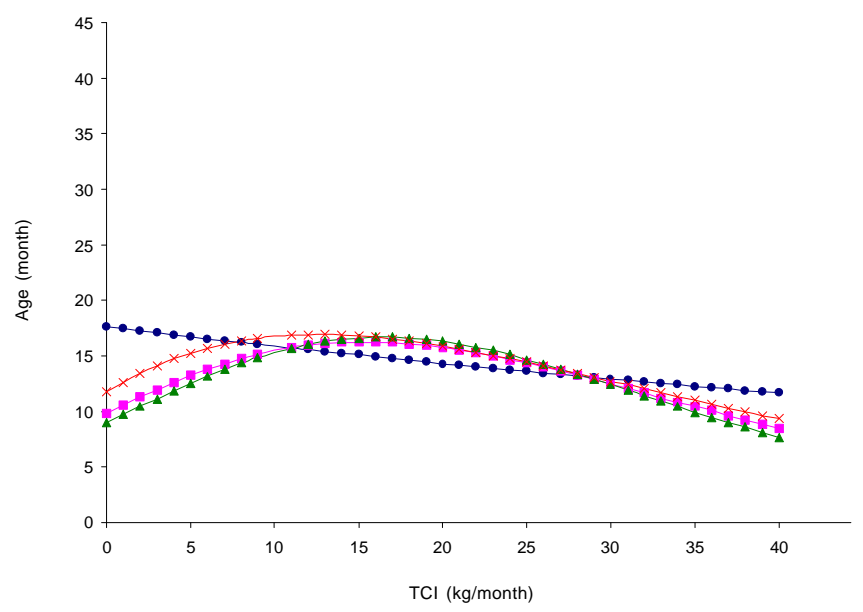

Figura 3 - Taxa de crescimento instantânea, em $\mathrm{kg} / \mathrm{mês}$ ( $\mathrm{TCl}, \mathrm{kg} / \mathrm{month}$ ), em função da idade (mês) de bovinos machos Canchim, obtidas dos modelos: 1) $\mathrm{y}_{1}=A\left(1-\right.$ be $\left.^{-k t}\right)$; 2) $\mathrm{y}_{2}=A \exp \left(-\right.$ be $\left.^{-k t}\right)$; 3) $\mathrm{y}_{3}=A$ / $(1+\exp (-k t))^{m}$ e 4) $y_{4}=A\left(1-b e^{-k t}\right)^{3}$, cujos índices são os mesmos dos gráficos.

Figure 3 - Instantaneous growth rate - TCl, $\mathrm{kg} /$ month, from male Canchim cattle obtained from the models: $y_{1}=A\left(1-b e^{-k t}\right) ; y_{2}=A \exp \left(-b e^{-k t}\right) ; y_{3}=A /(1+\exp (-k t))^{m}$ $e y_{4}=A\left(1-b e^{-k t}\right)^{3}$, whose indices are the same of the plots. de PI foram: $16,2 \mathrm{~kg}$ (16ํㅜ mês), $16,6 \mathrm{~kg}$ (16ํㅜ mês) e $16,0 \mathrm{~kg}$ (14으 mês), para os modelos Gompertz, Logístico e Von Bertalanffy, respectivamente.

No intervalo do peso ao nascimento $\left(\mathrm{t}_{\mathrm{i}}\right)$ e peso aos 40 meses de idade $\left(\mathrm{t}_{\mathrm{j}}\right)$, a taxa de crescimento média mensal, em kg, descrita no item i), para Brody, Gompertz, Logístico e Von Bertalanffy, foram, respectivamente, 13,$80 ; 12,99 ; 12,89$ e 13,22 . Por outro lado, a taxa de maturidade absoluta média, que indica a proporção de crescimento mensal relativa ao valor assintótico (A) foi, respectivamente, 0,$8 ; 1,7 ; 1,8 \mathrm{e}$ $1,5 \%$. Considerando-se que os pesos observados, em kg, aos 40 meses de idade foram 622,4; 610,0;609,9 e 613,9 para Brody, Gompertz, Logístico e Von Bertalanffy, respectivamente, e os correspondentes valores assintóticos (A): 1751,90; 780,59; 731,00 e $872,90 \mathrm{~kg}$, são necessários 126,8; 60,1; 56,7 e 66,0 meses de idade para se atingir o valor de A. O valor de $1,5 \%$ obtido para o modelo Von Bertalanffy, por exemplo, indica que a cada mês houve crescimento corporal de $1,5 \%$ relativo ao peso assintótico $(872,9 \mathrm{~kg})$, o que necessitaria 66,7 meses de idade para se atingir o peso à maturidade para a raça, caso o mesmo comportamento do crescimento se mantivesse após os 40 meses de idade. Considerando-se que a estimativa do peso ao nascimento é de $46,6 \mathrm{~kg}$, isto significa que o crescimento pós-nascimento é de $826,3 \mathrm{~kg}$, o que requer crescimento médio de $12,4 \mathrm{~kg} / \mathrm{mês}$.

Vários estudos sobre curvas de crescimento tem sido realizados em bovinos, tanto taurinos, quanto zebuínos e os resultados comprovam que, apesar de vários modelos serem adequados, existem divergências entre eles. Segundo Cortareli (1973), citado por Paz (2002), na comparação dos modelos Brody, Logístico, Richards, Von Bertalanffy e Gompertz, o último foi o mais adequado na estimação de crescimento de Nelore. Entretanto, conforme Perotto et al. (1992), o Richards foi o escolhido para estimar o crescimento em peso de fêmeas zebuínas e de mestiças Holandês x Zebu.

Para a avaliação de dados de fêmeas da raça Guzerá (Oliveira et al., 2000), os modelos Brody, Gompertz, Von Bertalanffy e Logístico apresentaram boa qualidade do ajuste, ao passo que o Von Bertalanffy, a melhor qualidade ajuste. O modelo Richards, por sua vez, apesar de ajustar bem os dados, apresentou dificuldades computacionais, não sendo indicado por esse autor para representar a curva de crescimento.

Elias (1998), no ajuste de dados de pesos de 
fêmeas Nelore, Guzerá e Gir, verificou que o modelos Brody, Logístico, Von Bertalanffy, Gompertz e Richards apresentaram bom ajuste aos dados; no entanto, o Brody foi o mais adequado. Para machos Nelore, pesados ao nascimento, ao desmame e mensalmente dos 10 aos 16 meses de idade, o modelo Von Bertalanffy apresentou melhor ajuste (Unanian et al., 2000). Mais recentemente (Paz, 2002), no ajuste dos modelos Brody, Von Bertalanffy, Richards, Gompertz e Logístico a dados de pesos ao nascimento, ao desmame e mensais dos 8 aos 19 meses de idade de 11 classes de genótipos e três grupos genéticos de bovinos Canchim, o último foi o mais indicado.

\section{Conclusões}

O modelo Logístico $\mathbf{y}=\mathbf{A} /\left(\mathbf{1}+\mathbf{e}^{-\mathbf{k t}}\right)^{\mathrm{m}}$, seguido do Von Bertalanffy $\mathbf{y}=\mathbf{A}\left(\mathbf{1}-\mathbf{b e}^{-\mathbf{k t}}\right)^{\mathbf{3}}$, foram os mais versáteis para ajustar dados de crescimento das espécies animais estudadas.

Os dois modelos Gompertz: $\mathbf{y}=\mathbf{y o .} \exp [(\mathbf{L} / \mathbf{K})(\mathbf{1}-$ $\left.\mathbf{e}^{-\mathbf{k t}}\right)$ ] e $\mathbf{y}=\mathbf{A} \cdot \exp \left(-\mathbf{b} \mathbf{e}^{-\mathbf{k t}}\right)$, foram adequados para predizer o crescimento de camarão, rã, coelho, frango, suíno e bovino.

De modo geral, modelos não-lineares tenderam a superestimar os pesos iniciais e finais em todas as espécies animais estudadas.

Em todas as espécies estudadas, pelo menos dois dos sete modelos não-lineares estudados mostraramse adequados para estimar o crescimento corporal, em função da idade, pois os coeficientes de determinação foram superiores a $92,0 \%$.

\section{Literatura Citada}

AGOSTINHO, C.A.; SILVA, M.A.; TORRES, R.B. et al. Curvas de crescimento de rãs-pimenta, Leptodactylus labyrinthicus (SPIX,1824). Revista da Sociedade Brasileira de Zootecnia, v.20, n.1, p.47-54, 1991.

BROWN, J.E.; FITZHUGH JR., H.A.; CARTWEIGHT,T.C A comparison of nonlinear models for describing weight-age relationship in cattle. Journal of Animal Science, v.42, n.4, p.810-818, 1976.

CARDOSO MONTEIRO, A.M.; AZEVEDO, J.M.T.; SILVA, E.S.R. Curvas de Crescimento de caprinos machos da raça Serrana Transmontana. Revista Portuguesa de Zootecncia, v.2, n.2, 1999. Disponível em: http://home.utad.pt/apez/ Publicacoes/revista/ano-v-2/art-04.htm. Acesso em: $21 \mathrm{de}$ jan. 2004.

DAVIDIAN, M.; GILTINAN. D.M. Nonlinear models for repeated measurement data. 2.ed. London: Chapman Hall, 1996. 359p.
DUTRA JR., W.M.; FERREIRA, S.; DONZELE, J.L. et al. Predição de curvas de crescimento de tecidos de fêmeas suínas por intermédio da função alométrica estendida. Revista Brasileira de Zootecnia, v.30, n.3, p.1007-1014, 2001 (suplemento 1).

DRAPER, N.R.; SMITH, H. Applied regression analysis. 2.ed. New York: Wiley, 1980. 709p.

ELIAS, A.M. Análise de curvas de crescimento de vacas das raças Nelore, Guzerá e Gir. Piracicaba: Escola Superior de Agricultura Luiz de Queiroz, 1998. 128p. Dissertação (Mestrado em Melhoramento Animal) - Escola Superior de Agricultura Luiz de Queiroz, 1998.

FITZHUGH JR., H.A. Analysis of growth curves and strategies for altering their shape. Journal of Animal Science, v.42, n.4, p.1036-1051, 1976.

FREITAS, A.R.; COSTA, C.N. Ajustamento de modelos não lineares a dados de crescimento de suínos. Pesquisa Agropecuária Brasileira, v.18, n.10, p.1147-54, out., 1983.

FREITAS, A.R.; ALBINO, L.F; MICHELAN FILHO, T. et al. Modelos de curvas de crescimento em frangos de corte. Pesquisa Agropecuária Brasileira, v.19, n.9, p.1057-1064, 1984.

MANSOUR, H.; JENSEN, E.L.; JOHNSON, L.P. Analysis of covariance structure of repeated measurements in holstein conformation traits. Journal of Dairy Science, v.74, n.8, p.2757-2766, 1991.

MENDES, P.P.; MARINS, M.A. Diferentes colunas de água no cultivo do camarão Macrobrachium rosenbergii (de Man, L. 879). Revista da Sociedade Brasileira de Zootecnia, v.24, n.6, p.863-873, 1995.

OLIVEIRA, H.N.L; LÔBO, R.B.; PEREIRA, C.S. Comparação de modelos não-lineares para descrever o crescimento de fêmeas da raça Guzerá. Pesquisa Agropecuária Brasileira, v.35, n.9, p.1843-1851, 2000.

PAZ, C.C.P.; PACKER, I.U.; FREITAS, A.R. et al. Ajuste de modelos não-lineares em estudos de associação entre polimorfismos genéticos e crescimento em bovino de corte. Revista Brasileira de Zootecnia, v.33, n.6, p.1416-1425, 2004.

PEROTTO, D.; CUE, R.I.; LEE, A.J. Comparison of nonlinear functions for describing the growth curve of three genotypes of dairy cattle. Canadian Journal of Animal Science, v.72, p.773-782, 1992.

RICHARDS, F.J.A. Flexible growth function for imprical use. Journal of Experimental Botany, v.10, n.29, p.290-300, 1959.

RODRIGUES, P.B.; MUNIZ, J.A.; PEREIRA, F.A. Estudo comparativo de curvas de crescimento em suínos. Ciência e Prática, v.16, n.1, p.151-157, 1992.

SANDLAND, R.L.; McGILCHRIST, C.A. Stochastic growth curve analysis. Biometrics, v.35, n.1, p.255-271, 1979.

SANTOS, C.L.; PEREZ, J.R.O; MUNIZ, J.A. et al. Desenvolvimento relativo dos tecidos ósseo, muscular e adiposo dos cortes da carcaça de cordeiros Santa Inês. Revista Brasileira de Zootecnia, v.30, n.2, p.487-492, 2001.

STATISTICAL ANALYSES SYSTEM - SAS. User's guide: statistics. version 8, v.2, Cary: 1999-2001.

UNANIAN, M.M.; BARRETO, C.C.; FREITAS, A.R. et al. Associação do polimorfismo do gene do hormônio de crescimento com a característica peso em bovinos da raça Nelore. Revista Brasileira de Zootecnia, v.29, n.5, p.380-386, 2000.

Recebido em: 06/01/04 Aceito em: 29/12/05 